\title{
Combined Topical and Systemic Administration with human adipose-derived mesenchymal stem cells (hADSC) and hADSC-derived exosomes markedly promoted cutaneous wound healing and regeneration
}

\section{Yang Zhou}

Tongji University School of Medicine https://orcid.org/0000-0002-6967-421X

\section{Bo Zhao}

Tongji University School of Life Science and Technology

Liao Xing Zhang

Tongji University School of Medicine

Jun Yi Lu

Tongji University School of Medicine

Tao Shou Lu

Tongji University School of Medicine

Jian Cheng

Tongji University School of Life Science and Technology

Yu Fu

Tongji University School of Science and Technology

Lin Lin

Tongji University School of Medicine

Yan Ning Zhang

Tongji University School of Life Science and Technology

Xin Pei Li

Tongji University School of Medicine

Jing Zhang

Tongji University School of Life Science and Technology

Jun Zhang ( $\boldsymbol{\nabla}$ junzhang@tongji.edu.cn )

Tongji University School of Life Science and Technology

Research

Keywords: Tongji University School of Life Science and Technology 
Posted Date: October 5th, 2020

DOl: https://doi.org/10.21203/rs.3.rs-85306/v1

License: (c) (i) This work is licensed under a Creative Commons Attribution 4.0 International License. Read Full License

Version of Record: A version of this preprint was published at Stem Cell Research \&amp; Therapy on May 1st, 2021. See the published version at https://doi.org/10.1186/s13287-021-02287-9. 


\section{Abstract}

Background: Cutaneous wound healing and regeneration has become a recognized health challenge in the world, which causes severe damage to the mental and physical health of patients. Human Adiposederived mesenchymal stem cells (hADSC) play an essential role in wound healing via their paracrine function. Exosomes secreted by hADSC may contribute to this progress. In this study, we investigated the potential clinical application roles of hADSC and hADSC-derived exosomes (hADSC-Exo) in cutaneous wound healing.

Methods: hADSC-Exo was isolated from human hADSC by ultracentrifugation. Mice were subjected to a full-thickness skin biopsy experiment and treated with either control vehicle or hADSC or hADSC-Exo by smearing administration (sm) or subcutaneous administration (sc) or intravenous administration (iv). The efficacy of hADSC and hADSC-Exo on wound healing was evaluated by measuring wound closure rates, histological analysis.

Results: Combined application of local hADSC-Exo smearing and hADSC/hADSC-Exo intravenous administration offered the additional benefit of promoting wound healing, accelerating reepithelialization, reducing scar widths, and enhancing angiogenesis and collagen synthesis. Either topical application of hADSC-Exo or systemic administration with hADSC/hADSC-Exo appeared more effective in stimulating cell proliferation, inhibiting cell apoptosis and inflammation, promoting skin elasticity and barrier integrity, with increased genes expression of PCNA, VEGF, collagen III, Filaggrin, Loricrin, and AQP3, with decreased genes expression of TNF-alpha.

Conclusion: Our findings suggest that the combined administration of hADSC/hADSC-Exo can facilitate cutaneous wound healing and reduce scar formation. These data provide the first evidence for the feasibility of smearing of hADSC-Exo as a cell-free therapy in treating cutaneous wounds, and the potential clinical value of combined administration of hADSC/hADSC-Exo.

\section{Full Text}

This preprint is available for download as a PDF.

\section{Figures}



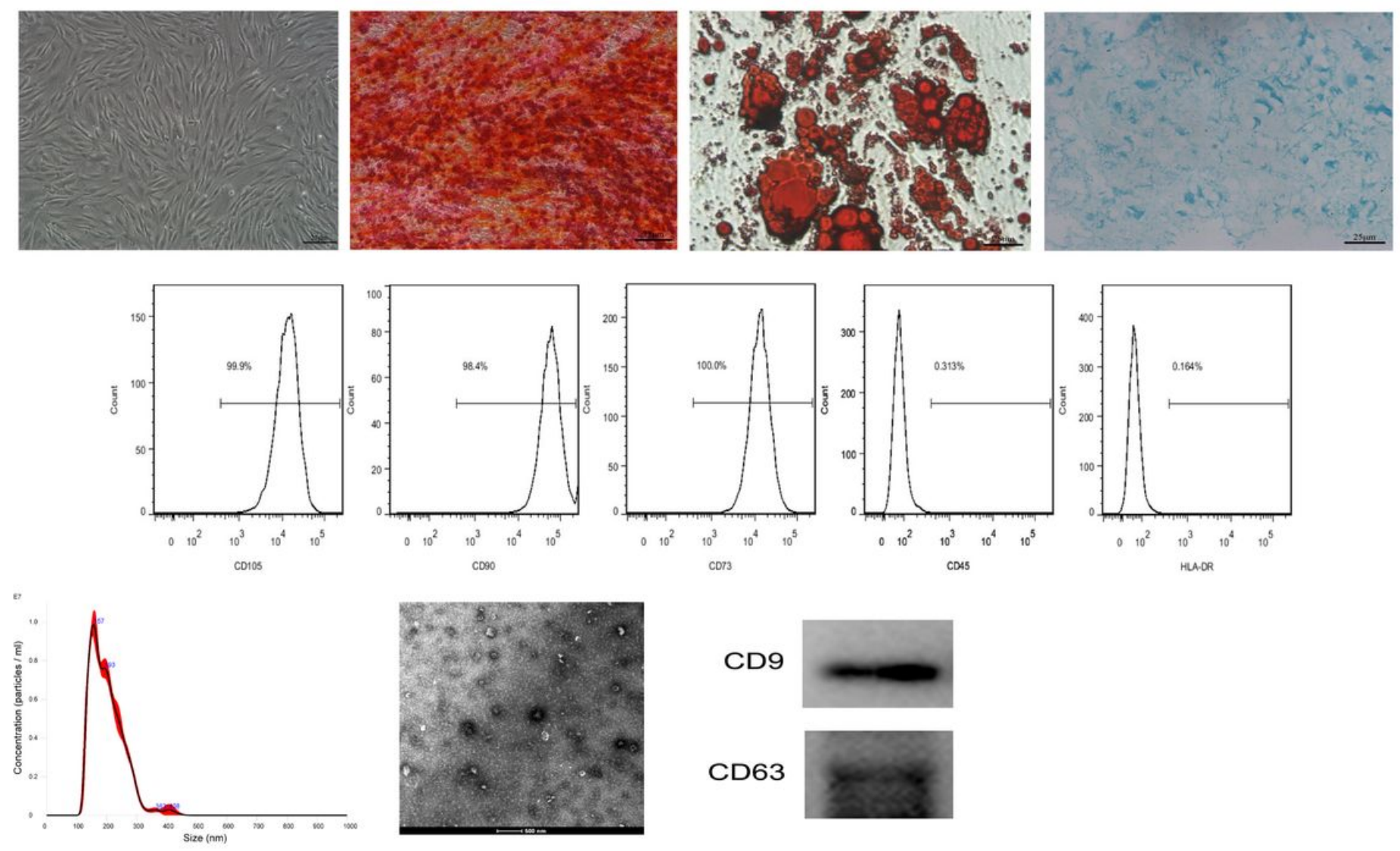

\section{Figure 1}

Isolation and characterization of hADSC and hADSC - exo. a Spindle hADSCs were observed through the microscope. Scale bar $=25 \mu \mathrm{m}$. b, c, d Adipogenic, osteogenic and chondrogenic differentiation of hADSCs. Cells differentiated into adipocyte, osteoblasts and chondrogenic were detected using Oil Red O, Alizarin Red and Alcian Blue, respectively. Scale bar $=25 \mu \mathrm{m}$. e Flow cytometry of hADSCs surface markers CD29, CD73, CD105, SSEA-3, SSEA-4 and HLA-DR. f Nanoparticle analysis of ADSC-exo. g TEM analysis of exosomes. Scale bars $=500 \mathrm{~nm}$. h Immunoblotting for CD9 and CD63 in exosomes. 

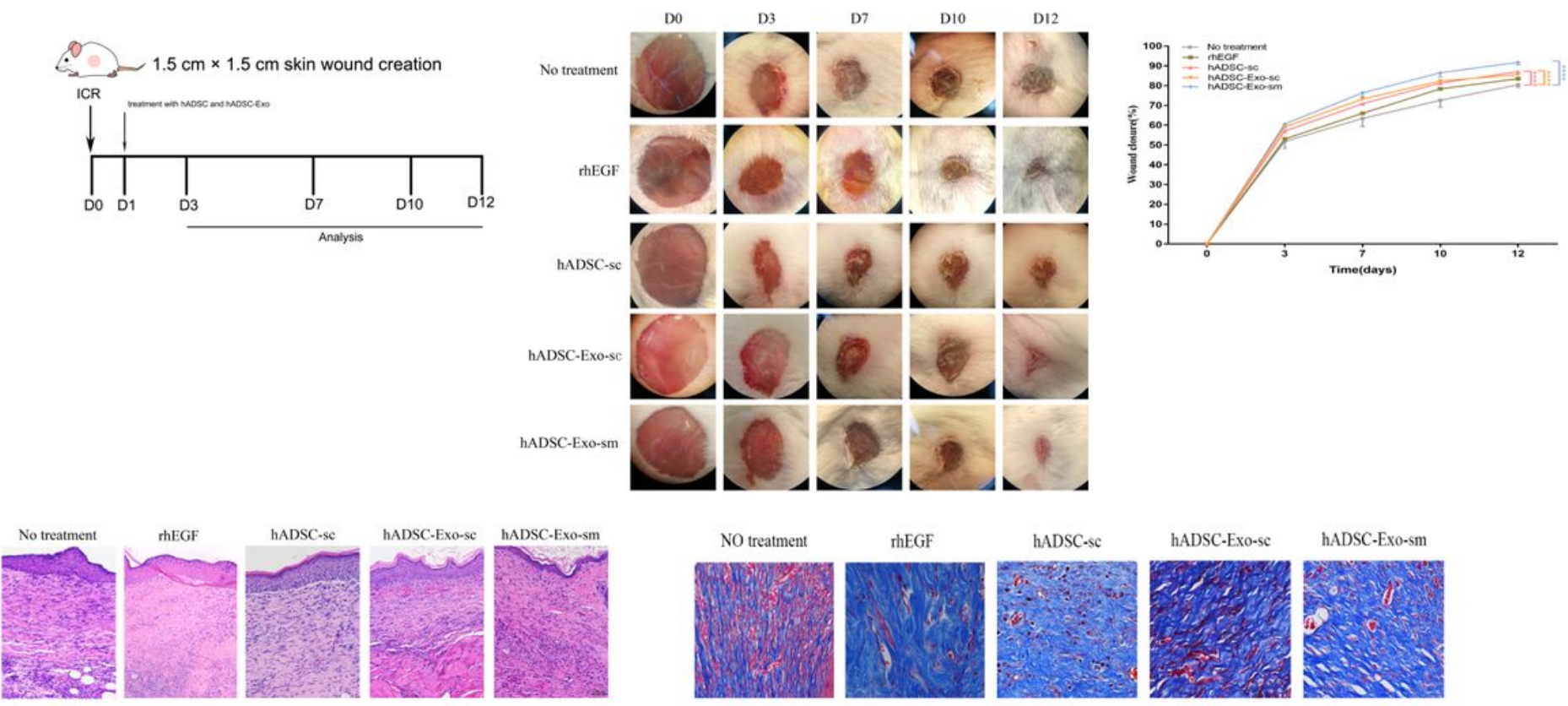

\section{Figure 2}

hADSC-Exo enhance cutaneous wound healing in an in vivo mouse model. a The study protocol. b Representative photographs of full-thickness excision wounds treated with rhEGF (positive control), hADSC or hADSC-Exo. hADSC-Exo were administered either by sm or sc for 12 days. c Quantitative analysis of wound healing in each group. $\mathrm{d}$ H\&E staining of wounded skin sections in different groups at day 12 post-wounding. Scale bar $=250 \mu \mathrm{m}$. e Masson staining of wounded skin sections in different groups at day 12 post-wounding. Results are presented as mean \pm standard error of the mean; $\mathrm{n}=8$ for each group. ${ }^{*} p<0.05,{ }^{*} p<0.01,{ }^{* \star} p<0.001$, and ${ }^{* \star * *} p<0.0001$ vs vehicle control group. sm smearing administration, sc subcutaneous administration. 

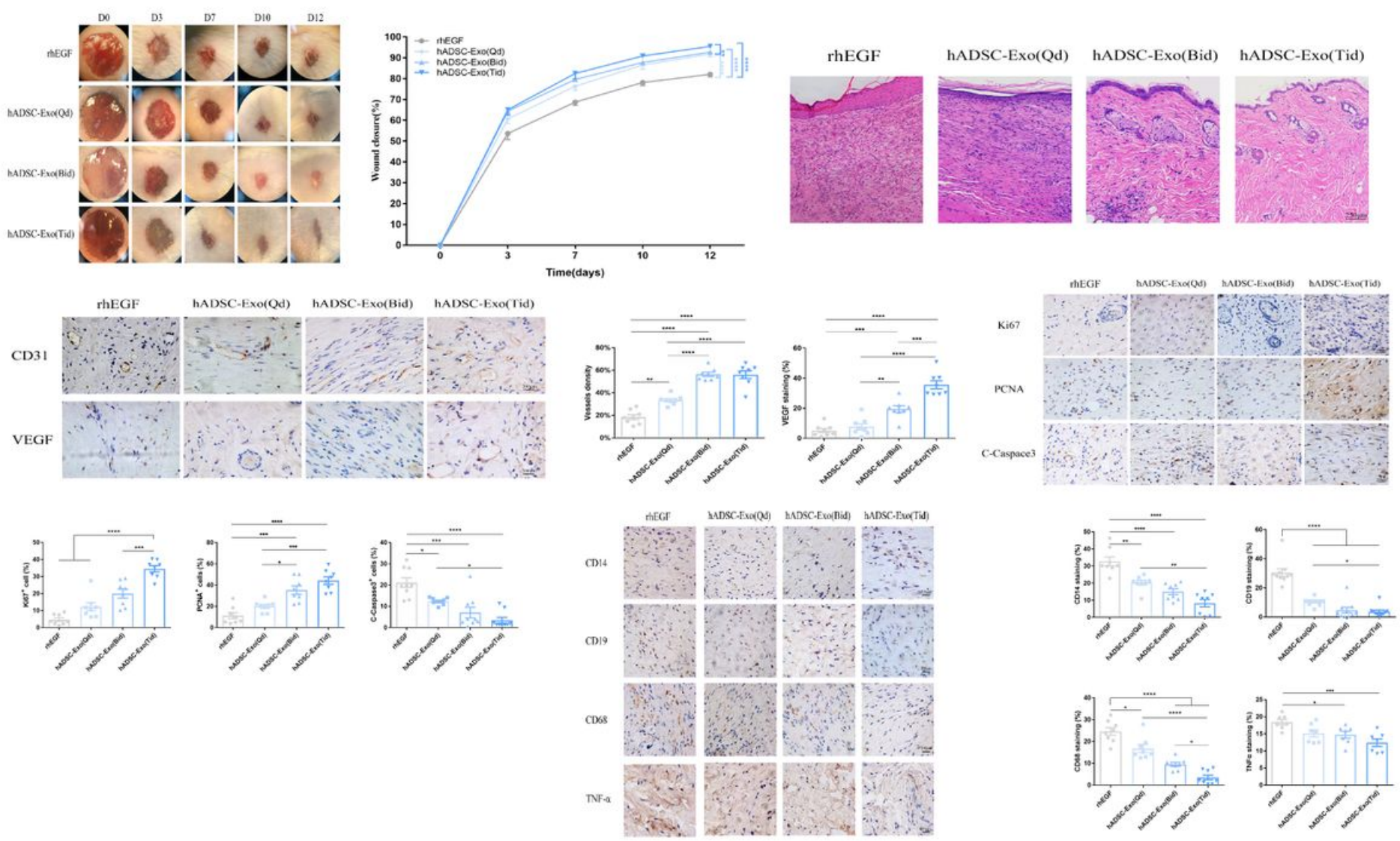

\section{Figure 3}

Effect of hADSC-Exo treatment on wound healing in vivo mouse model in a dose-dependent manner. a Representative photographs of full-thickness excisional wounds treated with hADSC-Exo (Qd); hADSC Exo (Bid); hADSC -Exo (Tid). b Quantitative analysis of wound healing in each group. c Histological structure of wounded skin in different groups. Scale bar $=250 \mu \mathrm{m}$. d Representative photographs of CD31 and VEGF immunostaining. Scale bar $=200 \mu \mathrm{m}$. e Quantitative analysis of the number of mature blood vessels. $\mathrm{f}$ Representative photographs of Ki67, PCNA and C-Caspase3 immunostaining. Scale bar $=$ $200 \mu \mathrm{m}$. g Ki67, PCNA and C-Caspase3 expression of IHC positive staining analyzed by mean percentage ( \pm SEM). h Representative photographs of CD14, CD19, CD68, MPO and TNF-alpha immunostaining. Scale bar $=200 \mu \mathrm{m}$. i Quantification of CD14+, CD19+, CD68+, and TNF-alpha+ IHC stained tissues. The rhEGF was used as a positive control. Results are presented as mean \pm standard error of the mean; $\mathrm{n}=8$ for each group. ${ }^{\star} p<0.05,{ }^{*} p<0.01,{ }^{* \star} p<0.001$, and ${ }^{\star \star \star \star} p<0.0001$ vs vehicle control group. 

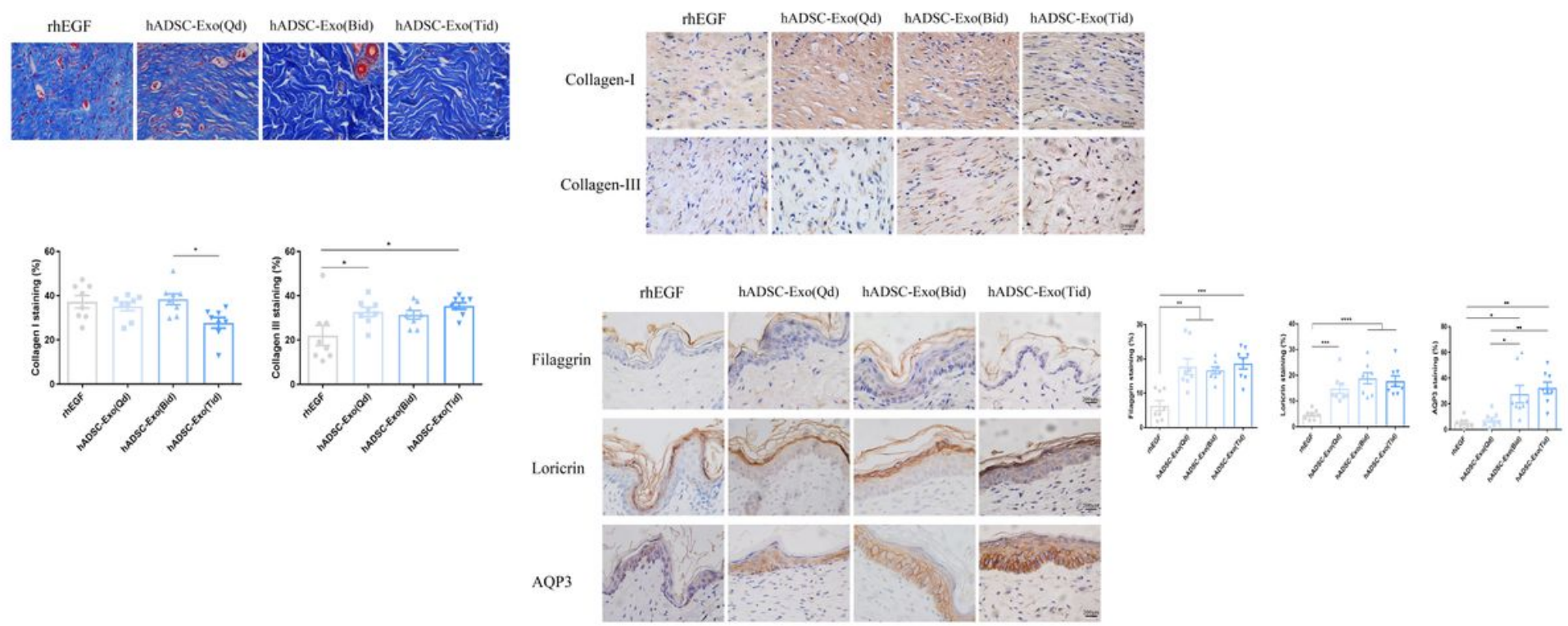

Figure 4

Histological assessment of scar after wound healing in different groups. a Masson staining of wounded skin sections in different groups at day 12 post-wounding. Scale bar $=200 \mu \mathrm{m}$. b Quantitative analysis of the number of collagenous fiber. c The results of immunohistochemical analysis of collagen I and collagen III were same as above. Scale bar $=200 \mu \mathrm{m}$. d Quantitative analysis of collagen I and collagen III. e immunohistochemical analyses were performed by using anti- Filaggrin, anti-Loricrin, and anti-AQP3 antibodies in skin wounds. Scale bar $=200 \mu \mathrm{m}$. $\mathrm{f}$ Quantification of Filaggrin, Loricrin, and AQP3 IHC stained tissues. The rhEGF was used as a positive control. Results are presented as mean \pm standard error of the mean; $\mathrm{n}=8$ for each group. ${ }^{*} \mathrm{p}<0.05,{ }^{\star \star} \mathrm{p}<0.01,{ }^{\star \star \star} \mathrm{p}<0.001$, and ${ }^{\star \star \star \star *} \mathrm{p}<0.0001$ vs vehicle control group. 

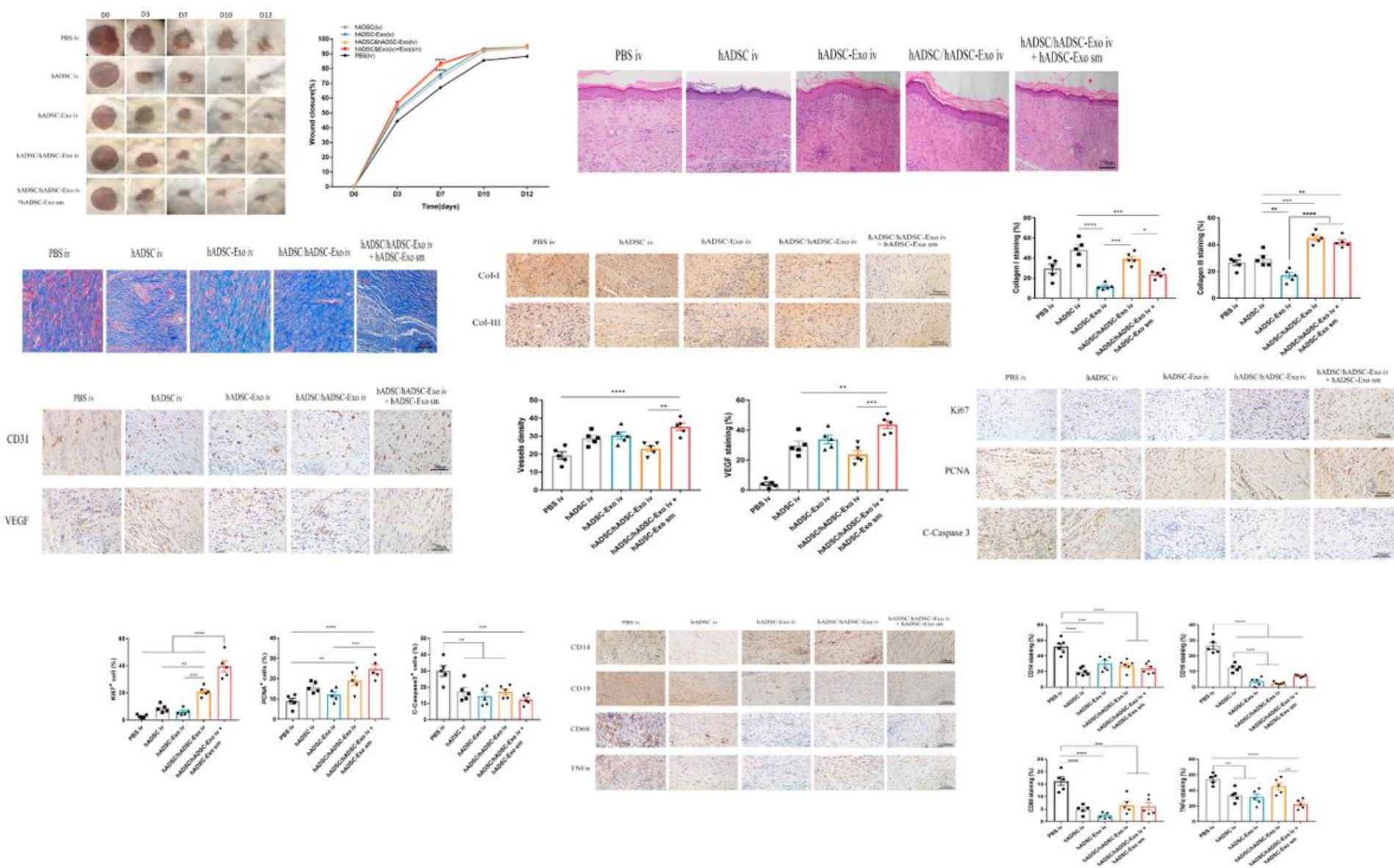

\section{Figure 5}

Therapeutic effects of hADSC-Exo in an in vivo mouse model. a Representative photographs of fullthickness excision wounds treated with hADSC iv and hADSC-Exo iv with or without hADSC-Exo sm. b Quantitative analysis of wound healing in each group. c Histological structure of wounded skin in different groups. Scale bar $=250 \mu \mathrm{m}$. d Masson staining of wounded skin sections in different groups at day 12 post-wounding. Scale bar $=200 \mu \mathrm{m}$. e The IHC of collagen-I and collagen-III in wounded skin sections on day 12 post-wounding. Scale bar $=200 \mu \mathrm{m}$. f Quantitative analysis of the number of Collagen deposition. g Representative images of immunohistochemical results of CD31 and VEGF. Scale bar $=200$ $\mu \mathrm{m}$. h Quantitative analysis of the number of mature blood vessels. Quantitative analysis of the positive cells in the membrane tissues. i Representative images of immunohistochemical results of Ki67, PCNA and C-Caspase3. Scale bar $=200 \mu \mathrm{m}$. j Quantitative analysis of the positive cells in the skin tissues. $\mathrm{k}$ Representative photographs of CD14, CD19, CD68, and TNF-alpha immunostaining. Scale bar $=200 \mu \mathrm{m}$. I Quantification of CD14+, CD19+, CD68+, and TNF-alpha+ IHC stained tissues. Results are presented as mean \pm standard error of the mean; $n=5$ for each group. ${ }^{\star} p<0.05,{ }^{\star \star} p<0.01,{ }^{\star} \mathrm{k} p<0.001$, and ${ }^{\star \star \star \star} \mathrm{p}<$ 0.0001 vs vehicle control group. 